\title{
On the Mean Motions Induced around a Planetary Wave Packet on a Rotating Sphere
}

\author{
By Michiya Uryu \\ Department of Physics, Faculty of Science, Kyushu University, Fukuoka, Japan. \\ (Manuscript received 19 October 1974, in revised form 20 December 1974)
}

\begin{abstract}
The mean motions induced around a planetary wave packet surrounding longitudinally a rotating sphere and propagating meridionally on it are discussed by using a simple model situation of two dimensional motion of an incompressible fluid.

It is shown that the wave packet conserves its angular momentum during the meridional propagation on a sphere, while it conserves its momentum under the beta-plane approximation. It is also shown that the wave packet induces the change of mean angular momentum in case of propagation on a sphere and the magnitude of the induced mean angular momentum is just equal to the wave action which is defined by the ratio of the wave energy and the westward drift angular velocity of the wave packet. Under the beta-plane approximation, this is reduced to the result that the induced mean zonal momentum is just equal to the wave momentum which is defined by the ratio of the wave energy and the 'local' westward phase velocity given by Rossby formula.

It is concluded from these results that the photon analogy to such a wave packet 'is allowed.

Finally, some comments are made upon the origin of westerlies found in the mid-latitudinal regions.
\end{abstract}

\section{Introduction}

Planetary-scale wave disturbances propagate upward and/or latitudinally far from their sources located mainly around the mid-latitudinal troposphere. They interact with the basic mean flow, and induce changes in it. Namely, they change the mean state of atmospheric rotation. Thus, it is not only of meteorological importance but also very interesting in a purely fluid dynamical viewpoint of wave-flow interaction to study how the planetary-scale disturbances affect the mean circulation of the atmosphere.

In his recent papers by the present author (Uryu, 1974a, b), it has been shown that a vertically propagating wave packet (Rossby wave or internal gravity wave) with infinite zonal length induces the second order mean zonal flow whose magnitude is just equal to the wave momentum $\mathrm{E} / \mathrm{C}$ (where $\mathrm{E}$ and $\mathrm{C}$ are the wave energy and the phase velocity in the zonal direction respectively). In other words, this result shows that the photon analogy to such waves, which is included implicitly in several works treating wave-flow interaction, such as Booker and Bretherton (1967), Lindzen and Holton (1968) and Matsuno (1971), is allowable; the easterly acceleration by an internal Rossby wave, for example, can be interpreted as a consequence of the wave packet possessing a negative (easterly) momentum and transferring it into the ambient mean zonal flow. In addition, according to this result, we can readily estimate the effect of planetary or internal gravity waves on the upper atmospheric mean circulation only if we know the wave energy and the phase velocity.

The emphasis in these works above is mainly focused on the interaction between lower and upper atmospheric layers. However, the lateral interaction between high and low latitudinal atmospheres is important as well. Especially, in relation to the penetration of planetary wave from the mid-latitude into the tropics and the resulting excitation of equatorial waves (Mak, 1969., for example), the interaction between a meridionally propagating planetary wave and the basic mean 
zonal flow has been studied by several authors; if the basic zonal flow is (assumed to be) constant in time, the wave is completely absorbed at the so-called critical level existing at the fringe of the tropical easterly region (Dickinson, 1970, Bennet and Young, 1971., for examples), while it can penetrate into the tropics if the basic zonal flow changes as a consequence of the wave absorption (Murakami, 1974).

Recently, Geisler and Dickinson (1974) have studied how a planetary wave propagating meridionally and incident on the critical level changes the basic mean zonal flow, and shown that the interaction continues until the wave becomes standing as a result of reflection brought about by the change of zonal mean vorticity gradient due to the wave absorption at the critical level.

The basic idea included implicitly in these works above is also the photon analogy that a meridionally propagating planetary wave possesses an easterly momentum and transfers it to the ambient mean zonal flow. As already shown (Uryu, 1974b), the photon analogy to a vertically propagating planetary or internal gravity wave holds validly under appropriate assumptions.

In the present paper, we shall examine and show the validity of the photon analogy for a planetary wave packet surrounding a rotating sphere and propagating meridionally on it.

In order to do so, we shall assume two dimensional motions of an incompressible fluid on a rotating sphere and use the same method (twotiming method) as in the previous paper (Uryu, 1974b), and then we shall discuss the second order mean motions induced around the wave packet. The results are summarized as follows.

In case of propagation on a beta-plane, the wave energy packed in the wave packet $E$ is conserved and the induced mean zonal momentum is just equal to the wave momentum $E / C$. This is similar to the result obtained in case of vertical propagation (Uryu, 1974b).

In case of propagation on a rotating sphere, the wave packet conserves the angular momentum Ecos $\theta / \omega$, where $\omega$ is the longitudinal drift angular velocity of the wave packet and $\theta$ is latitude. Thus, the wave packet induces the change in mean angular momentum, and the wave-induced mean angular momentum is just equal to $E / \omega$, which can be interpreted as the wave action of a planetary wave on a rotating sphere.
We should emphasize, however, that the waveinduced mean zonal flow inferred above exists only during the stay of the wave packet and vanishes if the wave propagates away. In this sense, the wave momentum is not 'deposited' to the mean flow if any diffusive process is not included. If viscous force acts on the propagating wave and destroys it, the wave momentum will be really transferred to the mean flow, to induce a kind of 'permanent motion' in it (cf. Bretherton, 1969).

We should further emphasize that the validity of the photon analogy shown above depends crucially upon the assumption that the wave packet surrounds the rotating sphere (or it is of infinite zonal length on a beta-plane). The assumption is often made in meteorological works, such as those cited above. However, if we remove the assumption, a different conclusion may be obtained (cf. McIntyre, 1973).

Furthermore, on the origin of westerlies found in the mid-latitudinal region, we shall make some comments, based upon the present results. On this problem, Rossby (1947) has already proposed a theory that the westerlies may be caused by the large-scale lateral mixing of absolute vorticity occuring in a polar cap and by the conservation of total angular momentum during the mixing process in the cap. His idea seems to be valid, at least, as the first approximation, from the viewpoint of the present paper.

\section{Two dimensional propagation on a beta-plane}

In this section, we shall discuss, as a preliminary consideration, the case of latitudinal propagation of planetary wave packet on a beta-plane.

The equation of two dimensional motion of an incompressible fluid on a beta-plane can be written in the form of vorticity equation as follows.

$$
\frac{\partial}{\partial t} \nabla^{2} \psi-\frac{\partial \psi}{\partial y} \frac{\partial}{\partial x} \nabla^{2} \psi+\frac{\partial \psi}{\partial x} \frac{\partial}{\partial y} \nabla^{2} \psi+\beta \frac{\partial \psi}{\partial x}=0,
$$

where $x$ and $y$ are the Cartesian coordinates in the zonal and the meridional directions respectively, $\phi$ is stream function, $\beta$ is the Rossby parameter and $\nabla^{2}$ stands for the horizontal Laplacian.

Here, we consider a wave packet having an infinite zonal length in the zonal direction and propagating in the latitudinal direction. The treatment in what follows is essentially similar to 
that used in the case of vertical propagation in the previous paper (Uryu, 1974b).

Separating $\phi$ into the wave packet part $\phi^{\prime}$ and the zonal mean part $\bar{\phi}$, i.e.,

$$
\phi=\phi^{\prime}+\bar{\phi}
$$

and introducing the slow variables as follows,

$$
Y=\epsilon y, \quad T=\epsilon t,
$$

where $\epsilon$ is a small parameter, we assume that $\phi^{\prime}$ has the following form.

$$
\psi^{\prime}=\operatorname{aRe}\left[\Psi(Y, T) e^{i(k x+l y+\sigma t)}\right],
$$

where $a$ is a small parameter characterizing the wave amplitude, $k$ and $l$ are the zonal and the meridional wave numbers respectively, $\sigma$ is the wave frequency and $\operatorname{Re}(\quad)$ means the real part of the quantity in the bracket. This assumption means that the wave packet has the predominant frequency $\sigma$, wave numbers $k$ and $l$, and that it has a slowly varying wave envelope $\Psi(Y, T)$ characterized by $\epsilon$.

$\bar{\psi}$ is assumed to be related to the induced mean zonal flow $\bar{U}$ as follows.

$$
\begin{aligned}
& \bar{\psi}=\bar{\psi}(y, Y, T), \\
& \bar{U}(Y, T)=-\frac{\partial \bar{\psi}}{\partial y} .
\end{aligned}
$$

These assumptions mean that, even if $\bar{\psi}$ itself varies with the fast variable $y$, its gradient varies only with the solw variable $Y$ (and $T$ ). Namely, the induced mean zonal flow varies with the same time and length scales as those of the wave envelope. It is noted that the wave packet part vanishes if it is averaged with respect to the fast varibles $x, y$ and $t$. Further, we assume that $\bar{\psi}$ is of second order in $a$.

In what follows, in addition to those assumptions above, we assume that the constancy of $\sigma$, $k$ and $l$. This is allowable in the present case, because the fluid system considered here is homogeneous geometrically as well as physically. However, as will be seen in the next section, the assumption of the constancy of $k$ and $l$ cannot be made on a rotating sphere, because the wave is contracted or elongated as it propagates in latitude. $\Psi, \bar{\psi}$ and $\bar{U}$ are expanded in $\epsilon$-series as follows.

$$
\begin{aligned}
& \Psi(Y, T)=\Psi_{0}+\epsilon \Psi_{1}+\cdots \cdots \\
& \bar{\psi}(y, Y, T)=\bar{\psi}_{0}+\epsilon \bar{\psi}_{1}+\cdots \cdots \\
& \bar{U}(Y, T)=\bar{U}_{0}+\epsilon \bar{U}_{1}+\cdots \cdots
\end{aligned}
$$

The other variables are also expanded in a similar manner, if necessary.

\section{(a) Wave packet solution}

Substituting (2-2) into (2-1) and picking up terms of 1 -st order in $a$, we obtain the following linearized vorticity equation.

$$
\frac{\partial}{\partial t} \nabla^{2} \psi^{\prime}+\beta \frac{\partial \psi^{\prime}}{\partial x}=0 .
$$

Substituting (2-4-a) into (2-6), we obtain, to 0 -th order in $\epsilon$, the following dispersion relation.

$$
\sigma=\frac{\beta k}{k^{2}+l^{2}} .
$$

Hence, the phase velocity in the zonal direction $C$ and the group velocity in the meridional direction $C_{g}$ become as

$$
C \equiv-\frac{\sigma}{k}=-\frac{\beta}{k^{2}+l^{2}},
$$

and

$$
C_{g}=-\frac{\partial \sigma}{\partial l}=\frac{2 \sigma l}{k^{2}+l^{2}}
$$

respectively.

From eq. (2-6), we obtain, to 1 -st order in $\epsilon$,

$$
\frac{\partial \Psi_{0}}{\partial T}+\frac{2 \sigma l}{k^{2}+l^{2}} \frac{\partial \Psi_{0}}{\partial Y}=0
$$

Eq. (2-9) can be readily rewritten by making use of $(2-8-b)$

$$
\frac{\partial \Psi_{0}}{\partial T}+C_{g} \frac{\partial \Psi_{0}}{\partial Y}=0
$$

Eq. (2-10) implies that the wave envelope does not change its shape during the propagation of the wave packet.

Here, we define the wave energy $E$ by

$$
E=\frac{1}{2} \rho\left[\left(\frac{\partial \psi^{\prime}}{\partial x}\right)^{2}+\left(\frac{\overline{\partial \psi}}{\partial y}\right)^{2}\right],
$$

where $\rho$ is the density of fluid and the bar means the zonal mean value with respect to the fast variable $x$.

Expanding $E$ in $\epsilon$-series, we have, to the leading order, 


$$
E_{0}=\frac{a^{2}}{4} \rho\left(k^{2}+l^{2}\right)\left|\Psi_{0}\right|^{2} .
$$

Thus, we can rewrite (2-10) in terms of $E_{0}$ as follows.

$$
\frac{\partial}{\partial T} E_{0}+C_{g} \frac{\partial}{\partial Y} E_{0}=0 .
$$

Eq. (2-13) means that the wave energy packed in the wave packet is conserved during the propagation. The same conclusion has been already obtained in case of vertical propagation (Uryu, 1974b). It should be noted here that eq. (2-13) holds only for a homogeneous medium. In an inhomogeneous but slowly varying medium, $E_{0}$ is replaced by $E_{0} / C$ and $C_{g}$ enters the operator $\frac{\partial}{\partial Y}$ in eq. (2-13) (cf. Bretherton and Garrett, 1969., for example). As will be shown in the next section, in case of propagation on a sphere, the conservation of wave angular momentum is obtained instead of eq. (2-13).

We calculate the Reynolds stress components. Using the following relationships

$$
u^{\prime}=-\frac{\partial \psi^{\prime}}{\partial y}, v^{\prime}=\frac{\partial \psi^{\prime}}{\partial x}
$$

where $u^{\prime}$ and $v^{\prime}$ are the zonal and meridional components of velocity perturbation respectively, we obtain, to leading order in $\epsilon$, that

$$
\begin{aligned}
& \rho \overline{u^{\prime} v^{\prime}}=C_{g} \frac{E_{0}}{C}, \\
& \rho \overline{v^{\prime 2}}=-\frac{k}{l} C_{g} \frac{E_{0}}{C} .
\end{aligned}
$$

\section{(b) Second order mean motoon}

We calculate the induced mean flow by the wave packet.

Substituting (2-2) into (2-1), averaging the result zonally with respect to the fast variable $x$ and taking (2-4-c) into account, we obtain an equation of the second order in $a$ as follows.

$$
\frac{\partial \bar{U}}{\partial t}-\frac{\partial}{\partial y}\left(\overline{\frac{\partial \psi^{\prime}}{\partial x} \frac{\partial \psi^{\prime}}{\partial y}}\right)=0 .
$$

This is also obtained directly by averaging the zonal component of equation of motion zonally, because the mean meridional current $\bar{V}$ vanishes identically due to continuity.

Substituting the wave packet solution (2-4-a) into the second term of $(2-15)$, we obtain, to leading order in $\epsilon$, that

$$
\frac{\partial\left(\rho \bar{U}_{0}\right)}{\partial T}=\frac{a^{2} \rho k l}{2} \frac{\partial\left|\Psi_{0}\right|^{2}}{\partial Y}=-\frac{\partial}{\partial Y}\left(C_{g} \frac{E_{0}}{C}\right) .
$$

Thus, making use of (2-13), we obtain one of the central results of this paper that

$$
\rho \bar{U}_{0}=\frac{E_{0}}{C} .
$$

This result means that the induced mean zonal momentum is just equal to the wave momentum, and hence the photon analogy is allowable also in the present case as in case of vertical propagation (Uryu, 1974b). It should be noted, however, that while, in the present case, the mean zonal momentum is induced by the momentum flux Reynolds stress), it is originated from the meridional buoyancy flux in the case of vertical propagation.

We should emphasize here that the wave-induced flow $\bar{U}$ inferred above exists only during the stay of the wave packet and vanishes if the wave propagates away. In this sense, the wave momentum $E / C$ is not 'deposited' or 'fixed' to the mean flow if any dissipative mechanism does not act on the wave to destroy it (cf. Bretherton, 1969). In a real fluid or atmosphere, wave momenta included in dissipated part of wave packet due to viscous force may be really transferred and deposited to the mean flow.

The structure of the induced mean flow fields in $y$-direction can be known from the meridional component of equation of motion. Averaging the equation zonally, we obtain

$$
\frac{\partial}{\partial y} \overline{v^{\prime 2}}+f \bar{U}=-\frac{1}{\rho} \frac{\partial \bar{P}}{\partial y},
$$

where $\bar{P}$ is the wave-induced zonal mean pressure. Note that $\bar{P}$ itself varies with fast variable $y$ but its gradient $\frac{\partial \bar{P}}{\partial y}$ is a function of the slow variables $Y$ and $T$ alone.

The substitution of (2-14) into the first term on the left of (2-18) shows that this term is one order in $\epsilon$ smaller than the other two terms. Hence, the induced mean zonal flow is in a geostrophic balance with the zonal mean pressure field, to leading order in $\epsilon$. Inversely speaking, the distribution of $\bar{P}$ in $y$-direction is determined 
so as to balance with the induced mean zonal flow $\bar{U}$ geostrophically.

\section{Two dimensional propagation on a rotating sphere}

Let $\theta$ and $\lambda$ be latitude and longitude respectively. Then, vorticity equation for two dimensional motion of an incompressible fluid on a rotating sphere (with unit radius) is written as

$$
\frac{\partial}{\partial t} \nabla^{2} \psi-\frac{\partial \psi}{\partial \theta} \frac{\partial \nabla^{2} \psi}{\cos \theta \partial \lambda}+\frac{\partial \psi}{\cos \theta \partial \lambda} \frac{\partial \nabla^{2} \psi}{\partial \theta}+2 \Omega \frac{\partial \psi}{\partial \lambda}=0,
$$

where $\Omega$ is the angular velocity of the rotating sphere, $\phi$ is the stream function which gives the zonal and the meridional velocity components, $u$ and $v$, through the following relationships,

$$
u=-\frac{\partial \psi}{\partial \theta}, v=\frac{1}{\cos \theta} \frac{\partial \phi}{\partial \lambda},
$$

and $\nabla^{2} \psi$ is defined as

$$
\nabla^{2} \psi=\frac{1}{\cos \theta} \frac{\partial}{\partial \theta}\left(\cos \theta \frac{\partial \psi}{\partial \theta}\right)+\frac{1}{\cos ^{2} \theta} \frac{\partial^{2} \psi}{\partial \lambda^{2}} .
$$

The treatment in what follows is similar to that in the previous section. One point to be noted is the effect of curvature which does not appear under the beta-plane approximation.

Introducing the slow variables $\Theta$ and $T$ defined as

$$
\Theta=\epsilon \theta, \quad T=\epsilon t
$$

we assume that the wave packet part of stream function $\psi^{\prime}$ and the zonal mean part $\bar{\psi}$ are written as

$$
\begin{aligned}
& \psi^{\prime}=a \operatorname{Re}\left[\Psi(\Theta, T) e^{i(s \lambda+l \theta+\sigma t)}\right], \\
& \bar{\psi}=\bar{\phi}(\theta, \Theta, T),
\end{aligned}
$$

where $s$ is the longitudinal mode number and $l$ is the latitudinal 'wave number' which is related to the latitudinal mode number $n$ as follows;

$$
l^{2} \cos ^{2} \theta=n^{2} \cos ^{2} \theta-s^{2} .
$$

This relation is verified, up to $0(1 / \mathrm{n})$, by the asymptotic expansion of Legendre function for large $n$ and small $s / n$ (Longuet-Higgins, 1964a). We see from (3-5-c) that a quasi-sinusoidal wave defined locally as (3-5-a) can exist only between two latitudes which satisfy $\cos \theta= \pm s / n$ (hereafter, we call these latitudes 'critical latitudes'(1) following Longuet-Higgins (1964a). The locations of critical latitudes depend on the value of $s / n$, and when it is small, are near the poles. In such a case, a quasi-sinusoidal form of wave can be reasonably assumed almost all over the spherical surface.

The longitudinal wave number $k$ is given by

$$
k=s \sec \theta \text {. }
$$

The mean zonal flow $\bar{U}$ is related to the zonal mean part $\bar{\psi}$ as follows;

$$
\bar{U}(\Theta, T)=-\frac{\partial \bar{\psi}}{\partial \theta} .
$$

Further, we assume that $\cos \theta$, appearing in eq. (3-1) and (3-5-c), is a slowly varying function of latitude $\theta$; that is,

$$
\cos \theta \equiv \cos \Theta, \quad \frac{\partial}{\partial \theta} \cos \theta \equiv-\epsilon \sin \Theta .
$$

Although a fluid on a rotating sphere is geometrically inhomogeneous (even if it is physically homogeneous), we can treat it as an almost homogeneous but slowly varying medium by the assumption (3-6). In addition, the wave numbers $k$ and $l$ are also slowly varying, and hence the length of the wave packet (3-5-a) in the latitudinal direction changes during the propagation. However, the total wave number is constant, i.e., $k^{2}+l^{2}=n^{2}$, as seen from (3-5-c) and (3-5-d). It is a characteristic property of the spherical harmonics that the total wave number is constant along a ray (Longuet-Higgins, 1964b).

According to the assumption (3-6), as will be shown in the subsection (a), the results consistent with those on the beta-plane but modified by the curvature are obtained.

\section{(a) Wave packet solution}

From eq. (3-1), we obtain, to 1 -st order in $a$, the following linearized vorticity equation.

$$
\frac{\partial}{\partial t} \nabla^{2} \psi^{\prime}+2 \Omega \frac{\partial \phi^{\prime}}{\partial \lambda}=0 \text {. }
$$

(1) When a wave propagates in a basic zonal flow with shear, a latitude where the phase velocity matches the zonal flow is often called 'critical latitude,' too. In order to avoid confusion, we call such a latitude 'critical level', if necessary, in this paper. 
Then, substituting (3-5-a) into (3-7), we have, to 0 -th order in $\epsilon$, the following dispersion relation.

$$
\sigma=\frac{2 \Omega s}{l^{2}+\frac{s^{2}}{\cos ^{2} \Theta}}=\frac{2 \Omega s}{n^{2}}
$$

Since $n^{2}$ is the total wave number and $s$ is related to $k$ through (3-5-d), we see that (3-8) is equivalent to (2-7) which is obtained on the beta-plane. It should be noted that, as seen from (3-8), $\sigma$ is constant along a ray (cf. Longuet-Higgins, 1964b).

If we define the westward drift angular velocity $\omega$ by $-\sigma / s$, it becomes as follows.

$$
\omega \equiv-\frac{\sigma}{s}=-\frac{2 \Omega}{n^{2}} .
$$

This is an approximation (with neglect to $1 / n$ ) of the angular velocity $2 \Omega / n(n+1)$ obtained in the exact treatment by spherical harmonics (cf. Longuet-Higgins, 1964a).

The 'local' phase velocity in the zonal direction is given by

$$
C=\omega \cos \Theta=-\frac{2 \Omega \cos \Theta}{n^{2}} .
$$

This is, of course, equivalent to the so-called Rossby formula. Thus, we see that, as already pointed out by Longeut-Higgins (1964a), the local beta-plane approximation given correct results with accuracy of $0(1 / n)$. by

The latitudinal group velocity $C_{g}$ can be given

$$
C_{g}=-\frac{\partial \sigma}{\partial l}=\frac{2 \sigma l}{n^{2}} .
$$

Then, it is seen that $C_{g}$ vanishes at the critical latitudes where $l=0$.

The group velocity $C_{g}{ }^{\prime}$ in the zonal direction becomes as

$$
C_{g}{ }^{\prime}=-\frac{\partial \sigma}{\partial k}=\frac{\sigma \cos \Theta\left(l^{2}-s^{2} / \cos ^{2} \Theta\right)}{n^{2} s}
$$

Thus, $C_{g}{ }^{\prime}$ does not vanish at the critical latitudes, and hence the ray path becomes parallel to the latitudinal circle there (Longuet-Higgins, 1964a). Since $l$ is imaginary outside the belt between the critical latitudes as seen from (3-5-c), the wave is totally reflected at the latitudes. Further, as seen from (3-5-c), the behavior of $l$ near a critical latitude $\theta_{0}\left(=\cos ^{-1} s / n\right)$ is as $l \propto\left(\theta-\theta_{0}\right)^{1 / 2}$, and hence it follows from (3-11-a) that $C_{g} \propto\left(\theta-\theta_{0}\right)^{1 / 2}$. Thus, the time taken for the wave packet to reach the critical latitude and be reflected is finite. In contrast, when a wave packet is incident on the so-called critical level where the phase velocity matches the basic zonal flow, it cannot reach the level for finite time duration, and in this sense the wave is absorbed there (Bretherton, 1966). The critical level is essentially different from the critical latitude in the present case.

From (3-10), (3-11-a) and (3-11-b), we readily obtain that

$$
C_{g}{ }^{2}+\left(C_{g}{ }^{\prime}+C\right)^{2}=\frac{4 \sigma^{2}}{n^{2}}
$$

This implies that the rate of progress of the wave packet along a ray rotating with the angular velocity $\omega$ is constant, as already mentioned by Longuet-Higgins (1964b).

From eq. (3-7), we obtain, to 1-st order in $\epsilon$, that

$$
\frac{\partial \Psi_{0}}{\partial T}+\frac{2 \sigma l}{n^{2}} \frac{\partial \Psi_{0}}{\partial \Theta}-\frac{\sigma l}{n^{2}} \tan \Theta \cdot \Psi_{0}+\frac{\sigma}{n^{2}} \frac{\partial l}{\partial \Theta} \Psi_{0}=0 .
$$

By making use of (3-11-a), eq. (3-12) can be written as

$$
\frac{\partial \Psi_{0}}{\partial T}+C_{g} \frac{\partial \Psi_{0}}{\partial \Theta}-\frac{C_{g}}{2} \tan \Theta \cdot \Psi_{0}+\frac{1}{2} \frac{\partial C_{g}}{\partial \Theta} \Psi_{0}=0 .
$$

Thus, the result (2-10) obtained on a beta-plane is extended to the case of a rotating sphere; the third term on the left of (3-13) shows the effect of curvature and the fourth shows that due to the change in the length of the wave packet in the latitudinal direction.

After some manipulation, (3-13) is reduced to the following equation;

$$
\frac{\partial}{\partial T}\left(\left|\Psi_{0}\right|^{2} \cos \Theta\right)+\frac{\partial}{\partial \Theta}\left(C_{g}\left|\Psi_{0}\right|^{2} \cos \Theta\right)=0,
$$

or

$$
\frac{\partial}{\partial T}\left(\left|\Psi_{0}\right|^{2} l \cos \Theta\right)+C_{g} \frac{\partial}{\partial \Theta}\left(\left|\Psi_{0}\right|^{2} l \cos \Theta\right)=0 .
$$

Eq. (3-14-b) states that $\left|\Psi_{0}\right|^{2} l \cos \Theta$ is constant along a ray or that the wave amplitude varies as

$$
\left|\Psi_{0}\right|^{2} \propto(l \cos \Theta)^{-1}
$$

along a ray. This is consistent with the result by Longuet-Higgins (1964b). It should be noted that 
(3-14-c) does not hold near the critical latitudes, in $\epsilon$, as follows.

where a quasi-sinusoidal wave form cannot be assumed. Further, we note that (3-14-b) means that the northward energy flux across any given latitudinal circle is constant (see below).

Here, defining the wave energy $E$ as,

$$
E=\frac{1}{2} \rho\left[\overline{\left(\frac{\partial \psi^{\prime}}{\partial \theta}\right)^{2}}+\frac{1}{\cos ^{2} \theta}\left(\overline{\left.\frac{\partial \psi^{\prime}}{\partial \lambda}\right)^{2}}\right],\right.
$$

we obtain, to leading order in $\epsilon$, that

$$
E_{0}=\frac{\rho}{4} a^{2} n^{2}\left|\Psi_{0}\right|^{2}
$$

Then, we can rewrite (3-14-a) and (3-14-b) as follows;

$$
\frac{\partial}{\partial T}\left(E_{0} \cos \Theta\right)+\frac{\partial}{\partial \Theta}\left(C_{g} E_{0} \cos \Theta\right)=0,
$$

or

$$
\frac{\partial}{\partial T}\left(E_{0} l \cos \Theta\right)+C_{g} \frac{\partial}{\partial \Theta}\left(E_{0} l \cos \Theta\right)=0 .
$$

Thus, it is readily seen by integrating (3-17-a) in a domain occupied by the wave packet that the total wave energy is conserved. Alternatively, (3-17-b) states that $E_{0} l \cos \Theta$ is conserved for an observer moving with the local group velocity. Further, we note that $E_{0} l \cos \Theta$ is proportional to the northward flux of wave energy across a given latitudinal circle.

Since $\omega$ is constant, (3-17-a) is equivalent to the following equation.

$$
\frac{\partial}{\partial T}\left(\frac{E_{0}}{\omega} \cos \Theta\right)+\frac{\partial}{\partial \Theta}\left(C_{g} \frac{E_{0}}{\omega} \cos \Theta\right)=0 .
$$

By making use of the relation $C=\omega \cos \Theta$, we can rewrite (3-18-a) as

$$
\frac{\partial}{\partial T}\left(\frac{E_{0}}{C} \cos ^{2} \Theta\right)+\frac{\partial}{\partial \Theta}\left(C_{g} \frac{E_{0}}{C} \cos ^{2} \Theta\right)=0 .
$$

Since $E_{0} / \omega$ is interpreted as the wave action, eq. (3-18-a) is the conservation law of angular momentum possessed by the wave packet. Eq. (3-18-b) is an alternative expression of the law in terms of the wave momentum $E_{0} / C$. Thus, the wave momentum conservation on a local betaplane is replaced with the conservation of wave angular momentum on a rotating sphere.

The Reynolds stress components associated with the wave packet become, to leading order

$$
\begin{aligned}
& \rho \overline{u^{\prime} v^{\prime}}=C_{g} \frac{E_{0}}{\omega \cos \Theta}=C_{g} \frac{E_{0}}{C}, \\
& \rho \overline{v^{\prime 2}}=-\frac{s}{n \cos \Theta} C_{g} \frac{E_{0}}{C} .
\end{aligned}
$$

\section{(b) Second order mean motion}

Averaging eq. (3-1) longitudinally with respect to the fast variable $x$, and taking (3-5-e) into account, we obtain the second order (in $a$ ) equation expressing the change in mean angular momentum as follows.

$$
\frac{\partial}{\partial t}(\bar{U} \cos \theta)-\frac{1}{\cos \theta} \frac{\partial}{\partial \theta}\left(\frac{\partial \psi^{\prime}}{\partial \lambda} \frac{\partial \psi^{\prime}}{\partial \theta} \cos \theta\right)=0 .
$$

This is obtained also directly by averaging the zonal component of equation of motion longitudinally and by taking into account that the mean meridional current vanishes identically due to continuity.

The substitution of the wave packet solution (3-5-a) into (3-20) gives, to leading order in $\epsilon$, that

$$
\frac{\partial}{\partial T}\left(\rho \bar{U}_{0} \cos \Theta\right)=-\frac{1}{\cos \Theta} \frac{\partial}{\partial \Theta}\left(C_{g} \frac{E_{0} \cos \Theta}{\omega}\right)
$$

Thus, making use of $(3-18-a)$ or $(3-18-b)$, we obtain that

$$
\rho \bar{U}_{0} \cos \Theta=\frac{E_{0}}{\omega}
$$

or

$$
\rho \bar{U}_{0}=\frac{E_{0}}{C}
$$

According to these results, the wave action $E / \omega$ induces the change of mean angular momentum, whose magnitude is just equal to $E / \omega$. Thus, it can be said that the photon analogy to a planetary wave packet on a rotating sphere is allowed as well as in case of propagation on a beta-plane; the wave packet possesses a welldefined wave action, transfers it to the ambient mean field and induces the change in mean angular momentum in it. However, as already mentioned in the previous section, it should be emphasized that viscous dissipation is required for the wave action or momentum to be really transferred and deposited to the mean flow.

The meridional structure of the induced mean 
flow fields can be known from the meridional component of equation of motion averaged longitudinally as follows.

$$
\begin{array}{r}
\left.\left\{\frac{1}{\cos \theta} \frac{\partial}{\partial \theta}\left[\overline{\left(v^{\prime 2}\right.}-\overline{u^{\prime 2}}\right) \cos \theta\right]+\frac{\partial}{\partial \theta} \overline{u^{\prime 2}}\right\} \\
-2 \Omega \sin \theta \vec{U}=-\frac{1}{\rho} \frac{\partial \bar{p}}{\partial \theta}
\end{array}
$$

The substitution of the wave packet solutions into the first term on the left of (3-23) shows that this term is one order in $\epsilon$ smaller than the other two terms, and hence a quasi-geostrophic balance holds between the mean zonal flow and the zonal mean pressure.

\section{Conclusions and remarks}

In the previous sections, by assuming two dimensional motions of an incompressible fluid on a rotating sphere, it has been shown that a planetary wave packet surrounding the sphere longitudinally and propagating meridionally conserves the wave angular momentum $E \cos \theta / \omega$ ( $E, \omega$ and $\theta$ being the wave energy, the westward drift angular velocity of the wave system and latitude respectively), and that the wave packet induces the change in mean angular momentum; $\rho \bar{U} \cos \theta=E / \omega(\bar{U}$ and $\rho$ being the induced mean zonal flow and the density of fluid respectively). Thus, $E / \omega$ can be interpreted as the action possessed by the wave packet and it is transferred to the ambient mean flow, to induce the change in mean angular momentum in it (photon analogy). Further, it has been also shown that, under the beta-plane approximation, the wave packet conserves the wave energy (and momentum) and induces the change in mean zonal momentum, i.e., $\rho \bar{U}=E / C(C$ being the 'local' phase velocity equal to $-\omega \cos \theta$ ).

We note, however, that the wave-induced flow exists only during the stay of the wave packet and vanishes if the wave propagates away. In this sense, the wave momentum or action is not deposited or fixed to the mean flow if any dissipative process is not included. In a real fluid or atmosphere, viscous force acts on the propagating wave, and hence momenta included in a dissipated part of the wave are really transferred and deposited to the mean flow; a kind of 'permanent motion' is induced (cf. Bretherton, 1969).

We should further emphasize that the validity of photon analogy depends crucially upon the zonally averaging process, i.e., the assumption that the wave amplitude envelope and hence the mean zonal flow are independent of longitude (cf. McIntyre, 1973). If we remove the assumption, such a simple result as the present one seems not to be obtained ${ }^{(1)}$.

Although we have not treated the case of propagation in a shear flow in this paper, we can speculate that, if we assume the shear flow $U$ to be a slowly varying function of latitude, the results summarized above may not be changed essentially except that $\omega$ or $C$ may be replaced with $U / \cos \theta-\omega$ or $U-C$ (cf. Uryu, 1974b). Thus, we can say that the critical level absorption of a planetary wave propagating meridionally on a rotating sphere (or on a beta-plane) occurs as a result that the wave action (or momentum) is stored up in the basic mean current, to induce the change of mean angular (or zonal) momentum in it. In other words, the momentum accumulation at the critical level is a consequence of infinitely long stay of the wave there, because the group velocity becomes infinitely small as the wave approaches near and near the critical level. The essential mechanism of such a critical level absorption that has been treated by Dickinson (1970) and Geisler and Dickinson (1974) is what has been described above.

The present results are very suggestive of the origin of westerlies found in the mid-latitudinal region. As has been mentioned above, a planetary wave packet propagating on a rotating sphere conserves its wave angular momentum and induces the change of mean angular momentum at its leading edge. Hence, when a planetary wave is excited at its source located around the midlatitude and propagates toward north and south, it accelerates easterly flows strongly in the northern side and weakly in the southern side of the wave source, and therefore there remain westerly flows near the wave source.

Then, as a result of strong acceleration of easterly flows, a critical level where the phase velocity of the wave matches the mean zonal flow will appear in the northern side of the wave source (cf. Matsuno, 1971), and after then a

(1) Further discussions will be mentioned elsewhere. According to his private communication from McIntyre, he develops a general theory on the photon analogy, though not yet been completed. 
process shown by Geisler and Dickinson (1974) will be realized; the critical level moves toward the wave source and the meridional gradient of zonal mean vorticity changes its sign there; then, the overreflection of wave begins to occur (cf. Jones, 1968., also) and the zonal flow configuration tends to become one which leads to total reflection of the incident wave; after then, the wave becomes standing, and the acceleration process ends. During the process above, the westerlies remaining near the wave source are maintained.

If viscous friction acts on the propagating wave, the wave-induced angular momentum will be deposited to the mean flow, as already mentioned above. Hence, if the wave continues to be excited, the westerlies will be maintained.

If the excited wave has its critical latitude (see §3) near its source, it is reflected totally there and cannot contribute to the production of westerlies. On the other hand, the critical latitudes of a wave with small longitudinal mode number (compared with the latitudinal mode number) are located near the poles (Longuet-Higgins, 1964a), and the contribution of such a wave may be large.

It should be remarked here that the above mentioned production process of westerlies in the mid-latitude is very similar to Rossby's idea proposed about 30 years ago (1947). He considered that the origin of the mid-latitudinal westerlies may be attributed to the large-scale lateral mixing of absolute vorticity which may occur in a polar cap, and in addition he mentioned that, as a result of the conservation of the total angular momentum in the polar cap during the mixing, strong westerlies appear at the boundary of the polar cap.

In the present paper, not a turbulent but a systematic transport of absolute vorticity by wave has been treated. Certainly, although it is questionable whether such a systematic transport can be called a mixing, we can consider that, if a planetary wave is so far from its source that it can be regarded as source-free, absolute vorticity may be 'diffused', being accompanied with particle dispersions due to the wave motion (Dickinson, 1969). Thus, if we regard the artificial boundary of the polar cap in Rossby's theory as a wave source, we see that there is no essential difference his theory and the present consideration. In this sense, Rossby's theory seems to be valid, at least, as a first approximation, although it seems to be disregarded nowadays.

The discussions mentioned above are qualitative, and a quantitative treatment by an appropriate method, such as a numerical experiment, is desired.

\section{Acknowledgements}

The author would like to express his sincere thanks to Prof. T. Matsuno, Tokyo University, for his many valuable suggestions and comments, stimulating discussions and continuing encouragement. The author is also indebted to Prof. R. Sawada, Kyushu University, for his valuable comments. Thanks are due to Dr. R. E. Dickinson, University of Colorado, Prof. J. R. Holton, University of Washington and Prof. M. E. McIntyre, University of Cambridge, for their kind advices and comments to improve the manuscript. This work was supported by Funds for Scientific Research of Ministry of Education.

\section{References}

Bennet, J. R. and J. A. Young, 1971: The influence of latitudinal wind shear upon large-scale wave propagation into the tropics. Mon. Wea. Rev. 99, 202-214.

Booker, J. R. and F. P. Bretherton, 1967: The critical layer for internal gravity waves in a shear flow. J. Fluid Mech., 27, 513-539.

Bretherton, F. P., 1969: On the mean motion induced by internal gravity waves. J. Fluid Mech., 36, 785802.

and C. J. R. Garrett, 1969: Wavetrains in inhomogeneous moving media. Proc. Roy. Soc. London, A, 302, 529-554.

Dickinson, R. E., 1969: Theory of planetaty wavezonal flow interaction. J. Atmos. Sci., 26, 73-81. 1970: Development of a Rossby wave critical level. J. Atmos. Sci., 27, 627-633.

Geisler, J. E. and R. E. Dickinson, 1974: Numerical study of an interacting Rossby wave and barotropic zonal flow near a critical level. J. Atmos. Sci., 31, 946-955.

Jones, W. L., 1968: Reflexion and stability of waves in stably stratified fluids with shear flow: a numerical study. J. Fluid. Mech., 34, 609-624.

Lindzen, R. S. and J. R. Holton, 1968: A theory of the quasi-biennial oscillation. J. Atmos. Sci., 25, 1095-1107.

Longuet-Higgins, M. S., 1964 a: Planetary waves on a rotating sphere. Proc. Roy. Soc. London. A, 279, 446-473.

, 1964 b: Planetary waves on a rotating sphere. II. Proc. Roy. Soc. London. A, 284, 40-68. 
McIntyre, M. E., 1973: Mean motions and impulse of a guided internal gravity wave packet. J. Fluid Mech., 60, 801-811.

Mak, M. K., 1969: Laterally driven stochastic motions in the tropics. J. Atmos. Sci., 26, 41-64.

Matsuno, T., 1971: A dynamical model of the stratospheric sudden warming. J. Atmos. Sci., 28, 14791494.

Murakami, M., 1974: Influence of mid-latitudinal planetary waves on the tropics under the existence of critical latitude. J. Meteor. Soc. Japan. 52, 261-
271.

Rossby, C. G. and collaborators, 1947: On the general circulation of the atmosphere in middle latitudes. Bull. Am. Met. Soc., 28, 255-280.

Uryu, M., 1974 a: Induction and transmission of mean zonal flow by quasi-geostrophic disturbances. $J$. Meteor. Soc. Japan, 52, 341-364.

, 1974 b: Mean zonal flows induced by a vertically propagating Rossby wave packet. $J$. Meteor. Soc. Japan, 52, 481-490.

\section{球面上を伝わるプラネタリー波のまわりに誘導される平均流について}

\section{瓜生道也}

九州大学 理学部 物理学教室

回転している球面上を，東西にぐるりとひとめぐりしたプラネタリー波束が，南北に伝わるとき，そのまわりに誘 導される平均流について, two-timing method を用いて議論する. 但し, 非圧縮性流体の 2 次元運動を仮定する.

まず, そのような波束は伝播過程で角運動量 $E \cos \theta / \omega(E$ は, 波のェネルギー, $\omega$ は波の東西方向の drift 角速 度, $\theta$ は緯度）を保存することが示される。.そしてこの波束によって誘導される 2 次の平均帯状流 $\bar{U}$ は, $\rho \bar{U} \cos \theta$ $=E / \omega$ で与えられることがわかる ( $\rho$ は流体の密度). これらの結果は, ベータ平面上では, 波束が運動量 $E / C(C$ は 東西位相速度. $C=\omega \cos \theta)$ を保存し, 平均帯状流は $\rho \bar{U}=E / C$ で与えられると言いか学られる.

以上の結果から，この種の波束は，フォトン・アナロジーによって理解できることがわかる. 